\title{
A DERIVED EQUIVALENCE FOR A DEGREE 6 DEL PEZZO SURFACE OVER AN ARBITRARY FIELD
}

\author{
M. BLUNK, S.J. SIERRA, AND S. PAUL SMITH
}

\begin{abstract}
Let $S$ be a degree six del Pezzo surface over an arbitrary field $F$. Motivated by the first author's classification of all such $S$ up to isomorphism [3] in terms of a separable $F$-algebra $B \times Q \times F$, and by his K-theory isomorphism $K_{n}(S) \cong K_{n}(B \times Q \times F)$ for $n \geq 0$, we prove an equivalence of derived categories$$
\mathrm{D}^{b}(\operatorname{coh} S) \equiv \mathrm{D}^{b}(\bmod A)
$$

where $A$ is an explicitly given finite dimensional $F$-algebra whose semisimple part is $B \times Q \times F$.
\end{abstract}

\section{INTRODUCTION}

We will work over an arbitrary field $F$.

Throughout $S$ denotes a degree six del Pezzo surface over $F$. Equivalently, $S$ is a smooth projective surface over $F$ whose anti-canonical sheaf is ample and has self-intersection number 6 .

Throughout $\bar{F}$ will denote a separable closure of $F$ and we will write

$$
\bar{S}=S_{\bar{F}}=S \times_{\operatorname{Spec} F} \operatorname{Spec} \bar{F} .
$$

In [3, the first author classified such $S$ up to isomorphism by associating to $S$ a pair of $F$-algebras $B$ and $Q$, both defined as endomorphism rings of certain locally free sheaves on $S$. Furthermore, it was shown there that the algebraic K-theory of $S$ is isomorphic to that of the algebra $B \times Q \times F$.

Let $\operatorname{coh} S$ denote the category of coherent sheaves on $S$ and let $\bmod A \operatorname{denote}$ the category of noetherian right $A$-modules. Let $\equiv$ denote equivalence of derived categories. Our main result (Theorem 4.5) establishes a derived equivalence

$$
\mathrm{D}^{b}(\operatorname{coh} S) \equiv \mathrm{D}^{b}(\bmod A)
$$

where $A$ is a finite dimensional $F$-algebra whose semi-simple quotient is $B \times Q \times F$. We prove this equivalence by constructing a tilting bundle $\mathcal{T}$ on $S$ that has $A$ as its endomorphism ring. The main novelty of our approach is that we do not make any assumptions on the base field $F$. Since the field $F$ is arbitrary, we cannot assume that $S$ is obtained by blowing up $\mathbb{P}_{F}^{2}$ (in fact $S$ could be a minimal surface), nor can we use exceptional collections.

Acknowledgments. All three authors acknowledge the support of the National Science Foundation with gratitude. This research was partially done while the

1991 Mathematics Subject Classification. 116E35, 14F05, 14J26, 14J60.

Key words and phrases. Derived equivalence, del Pezzo surface, arbitrary field.

M. Blunk was supported by the National Science Foundation, Award No. 0902659. S.J. Sierra was supported by the National Science Foundation, Award No. 0802935. S.P. Smith was supported by the National Science Foundation, Award No. 0602347. 
first author was visiting the University of Washington, and he would like to thank the insitution for its support and excellent working conditions. Finally, the first author would like to thank Aravind Asok, Baptise Calmès, and Daniel Krashen for suggesting this problem.

\section{BASIC FACTS ABOUt $\bar{S}$}

In this section, we give basic facts about the degree 6 del Pezzo surface $\bar{S}$. Since all the results here are well-known, we do not give references.

There are six $(-1)$-curves on $\bar{S}$, which we may take to lie in the following configuration:

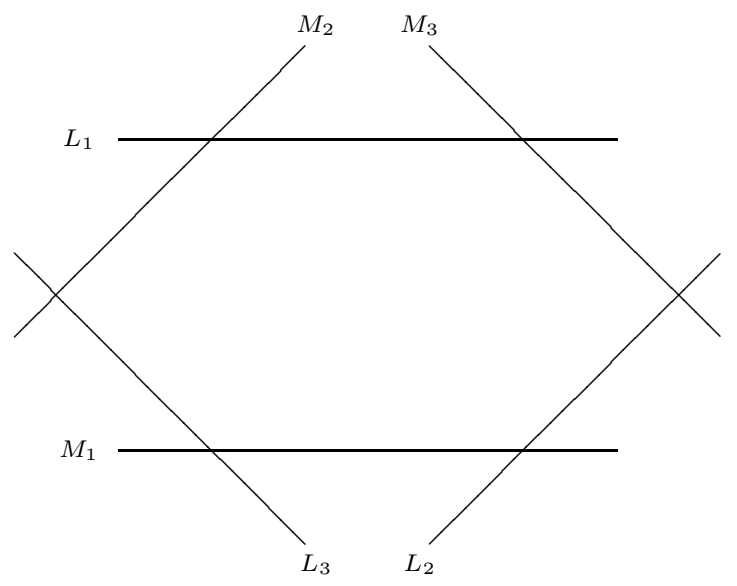

The Picard group is

$$
\operatorname{Pic} \bar{S} \cong \frac{\bigoplus_{i=1}^{3}\left(\mathbb{Z} L_{i} \oplus \mathbb{Z} M_{i}\right)}{\left(M_{i}+L_{j}=M_{j}+L_{i} \mid 1 \leq i, j \leq 3\right)} .
$$

Usually we only care about the class of a divisor in $\mathrm{Pic} \bar{S}$. We will write

$$
D_{1} \sim D_{2}
$$

if $D_{1}$ and $D_{2}$ are linearly equivalent divisors.

As remarked in the discussion after Prop. 2.1 in [3], the group of connected components of the group Aut $\bar{S}$ is $S_{2} \times S_{3}$, which can be identified with the automorphism group of the hexagon of $(-1)$-curves on $\bar{S}$. In particular, there is an element $\sigma \in \operatorname{Aut}(\bar{S})$ that cyclicly permutes the six exceptional lines. It is easy to see that $(1+\sigma)\left(1-\sigma^{3}\right)$ acts trivially on $\operatorname{Pic} \bar{S}$.

An anti-canonical divisor is

$$
-K_{\bar{S}}:=L_{1}+L_{2}+L_{3}+M_{1}+M_{2}+M_{3} .
$$

This is ample. We define two particular divisors

$$
H:=L_{1}+M_{2}+M_{3} \sim L_{2}+M_{1}+M_{3} \sim L_{3}+M_{1}+M_{2}
$$

and

$$
H^{\prime}:=L_{1}+L_{2}+M_{3} \sim L_{2}+L_{3}+M_{1} \sim L_{3}+L_{1}+M_{2}
$$

on $\bar{S}$. Note that $\sigma(H) \sim H^{\prime}$ and $\sigma^{2}(H) \sim H$.

We define the degree of a divisor $C$ on $\bar{S}$ as $\operatorname{deg} C=-C \cdot K$. Each exceptional line has degree 1 . 
There are two morphisms $f, f^{\prime}: \bar{S} \rightarrow \mathbb{P}_{\bar{F}}^{2}$, each of which realizes $\bar{S}$ as the blowup of $\mathbb{P}_{\bar{F}}^{2}$ at three non-collinear points. We choose these so that $f$ contracts the lines $L_{1}, L_{2}$, and $L_{3}$ and $f^{\prime}$ contracts the lines $M_{1}, M_{2}$, and $M_{3}$. These two morphisms induce injective group homomorphisms $f^{*}, f^{\prime *}: \operatorname{Pic} \mathbb{P}^{2} \rightarrow \operatorname{Pic} \bar{S}$. If $\ell$ is a line on $\mathbb{P}_{\bar{F}}^{2}$, then $f^{*} \ell=H$ and $f^{*} \ell=H^{\prime}$.

The action of $\operatorname{Gal}(\bar{F} / F)$ on the exceptional lines on $\bar{S}$ induces actions of $\operatorname{Gal}(\bar{F} / F)$ on

$$
\overline{\mathcal{I}}:=\bigoplus_{i=0}^{5} \mathcal{O}_{\bar{S}}\left(\sigma^{i} H\right)
$$

and

$$
\overline{\mathcal{J}}:=\bigoplus_{i=0}^{5} \mathcal{O}_{\bar{S}}\left(\sigma^{i}\left(L_{1}+M_{2}\right)\right)
$$

that are compatible with its action on $\bar{S}$. In particular, $\overline{\mathcal{I}}$ and $\overline{\mathcal{J}}$ are $\operatorname{Gal}(\bar{F} / F)$ invariant. It follows that $\overline{\mathcal{I}}$ and $\overline{\mathcal{J}}$ descend to sheaves $\mathcal{I}$ and $\mathcal{J}$ on $S$.

Define

$$
\overline{\mathcal{T}}:=\overline{\mathcal{I}} \oplus \overline{\mathcal{J}} \oplus \mathcal{O}_{\bar{S}}, \quad \mathcal{T}:=\mathcal{I} \oplus \mathcal{J} \oplus \mathcal{O}_{S}
$$

and

$$
B:=\operatorname{End}_{S} \mathcal{I}, \quad Q:=\operatorname{End}_{S} \mathcal{J}, \quad A:=\operatorname{End}_{S} \mathcal{T} .
$$

In $[3$ it is shown that $S$ is determined up to isomorphism by the pair of $F$-algebras $(B, Q)$. (Actually, in [3], $B$ is defined as $\left(\operatorname{End}_{S} \mathcal{I}^{\vee}\right)^{\mathrm{op}}$. Since sending a homomorphism $\alpha: \mathcal{I} \rightarrow \mathcal{I}$ to its transpose $\alpha^{\vee}: \mathcal{I}^{\vee} \rightarrow \mathcal{I}^{\vee}$ is an anti-isomorphism from $\operatorname{End}_{S} \mathcal{I}$ to $\operatorname{End}_{S} \mathcal{I}^{\vee}$ so our $B$ is the same as that in [3], and similarly for Q.) As discussed in [3], the algebras $B$ and $Q$ are Azumaya over their centers, which are respectively étale quadratic and cubic extensions of $F$. Moreover, these étale centers can be recovered from the action of $\operatorname{Gal}(\bar{F} / F)$ on the hexagon of $(-1)$-curves, as the action induces a 1-cocyle of $\operatorname{Gal}(\bar{F} / F)$ with values in $S_{2} \times S_{3}$, inducing a pair of étale extensions of $F$, quadratic and cubic.

To end this section, we give two results about the endomorphism algebra of $\mathcal{T}$.

Lemma 2.1. Let $A:=\operatorname{End}_{S} \mathcal{T}$. Then

$$
A=\left(\begin{array}{ccc}
B & \operatorname{Hom}(\mathcal{J}, \mathcal{I}) & \operatorname{Hom}\left(\mathcal{O}_{S}, \mathcal{I}\right) \\
0 & Q & \operatorname{Hom}\left(\mathcal{O}_{S}, \mathcal{J}\right) \\
0 & 0 & F
\end{array}\right) .
$$

Proof. It suffices to show $\operatorname{Hom}_{\bar{S}}(\overline{\mathcal{I}}, \overline{\mathcal{J}})=\operatorname{Hom}_{\bar{S}}\left(\overline{\mathcal{I}}, \mathcal{O}_{\bar{S}}\right)=\operatorname{Hom}_{\bar{S}}\left(\overline{\mathcal{J}}, \mathcal{O}_{\bar{S}}\right)=0$. However, each of these three Hom-spaces is isomorphic to a direct sum of terms of the form $H^{0}\left(\bar{S}, \mathcal{O}_{\bar{S}}(D)\right)$ for a divisor $D$ with $\operatorname{deg} D<0$. But if $D$ has a section then $D \sim D^{\prime}$ for some effective $D^{\prime}$ so $\operatorname{deg} D=-D^{\prime} . K \geq 0$. These Hom spaces are therefore zero.

The projective dimension of a left $T$-module is denoted by $\operatorname{pdim}_{T} M$. The global homological dimension of $T$ is defined and denoted by

$$
\operatorname{gldim} T:=\sup \left\{\operatorname{pdim}_{T} M \mid M \in \operatorname{Mod} T\right\} .
$$

Proposition 2.2. gldim $A \leq 2$. 
Proof. Let $R$ and $S$ be rings and $X$ an $R$-S-bimodule. If $S$ is a semisimple ring, then

$$
\operatorname{gldim}\left(\begin{array}{cc}
R & X \\
0 & S
\end{array}\right)=\max \left\{\operatorname{pdim}_{R} X+1, \operatorname{gldim} R\right\} .
$$

(See [1, Prop. III.2.7].) Applying this result twice, first to

$$
A^{\prime}:=\left(\begin{array}{cc}
B & \operatorname{Hom}(\mathcal{J}, \mathcal{I}) \\
0 & Q
\end{array}\right)
$$

then to $A$ with $R=A^{\prime}$ and $S=F$, gives the desired result.

\section{Cohomology VANishing Lemmas}

We will prove several results about vanishing of cohomology and Ext-groups for sheaves on $S$. These results will be used in Section 4 to show that $\mathcal{T}$ is a tilting bundle and therefore induces an equivalence of derived categories.

A key step in proving that $\mathcal{T}$ is tilting is showing that $\operatorname{Hom}_{S}^{i}(\mathcal{T}, \mathcal{T})=0$ for $i>0$. This reduces, by flat base change, to proving that $\operatorname{Hom}_{\bar{S}}^{i}(\overline{\mathcal{T}}, \overline{\mathcal{T}})=0$. Given the explicit description of $\overline{\mathcal{T}}$ as a direct sum of invertible sheaves, it suffices to prove that $h^{1}\left(D-D^{\prime}\right)=h^{2}\left(D-D^{\prime}\right)=0$ for all $D$ and $D^{\prime}$ belonging to the list

$$
H, \quad H^{\prime}, \quad L_{1}+M_{2}, \quad L_{2}+M_{3}, \quad L_{3}+M_{1}, \quad 0 .
$$

We will make repeated use of the relation $L_{i}+M_{j} \sim L_{j}+M_{i}$.

Proposition 3.1. Let $D$ and $D^{\prime}$ be divisors on $\bar{S}$ appearing in the list (3-1). Then

$$
-3 \leq \operatorname{deg}\left(D-D^{\prime}\right) \leq 3 .
$$

Furthermore,

(1) if $\operatorname{deg}\left(D-D^{\prime}\right)=1$, then $D-D^{\prime}$ is linearly equivalent to an exceptional line.

(2) if $\operatorname{deg}\left(D-D^{\prime}\right)=2$, then $D-D^{\prime} \sim L_{i}+M_{j}$ for some $i \neq j \in\{1,2,3\}$.

(3) if $\operatorname{deg}\left(D-D^{\prime}\right)=3$, then $D-D^{\prime}$ is linearly equivalent to either $H$ or $H^{\prime}$.

(4) if $\operatorname{deg}\left(D-D^{\prime}\right)=0$, then $D-D^{\prime}$ is linearly equivalent to either $0, L_{i}-L_{j}$, $L_{i}-M_{i}$, or $M_{i}-L_{i}$ for some $i, j \in\{1,2,3\}$.

(5) if $\operatorname{deg}\left(D-D^{\prime}\right)<0$, then $D-D^{\prime}$ is linearly equivalent to either $-L_{i}$, or $-M_{j}$, or $-L_{i}-M_{j}$, or $-H$, or $-H^{\prime}$.

Proof. Exceptional lines have degree 1 so $\operatorname{deg} H=\operatorname{deg} H^{\prime}=3$ and $\operatorname{deg}\left(L_{i}+M_{j}\right)=$ 2. It follows that the degree of $D-D^{\prime}$ is between 3 and -3 .

(1) If $\operatorname{deg}\left(D-D^{\prime}\right)=1$, then $D$ is linearly equivalent to $H$ or $H^{\prime}$ and $D^{\prime}=L_{i}+M_{j}$ for some $i, j$. It follows from (2-2) and (2-3) that $D-D^{\prime}$ is linearly equivalent to an exceptional line, and every exceptional line can occur as $D-D^{\prime}$.

(2) and (3) are obvious.

(4) In this case $D$ and $D^{\prime}$ have the same degree.

If $\operatorname{deg} D=\operatorname{deg} D^{\prime}=2$, then $D=L_{i}+M_{j}$ and $D^{\prime}=L_{k}+M_{\ell}$. By considering all possible $i, j, k, \ell$, we see that $D-D^{\prime}$ is linearly equivalent to a divisor of the form $L_{i}-L_{j}$.

If $\operatorname{deg} D=\operatorname{deg} D^{\prime}=3$, then, for example, $D \sim H$ and $D^{\prime} \sim H^{\prime}$, and $D-D^{\prime} \sim$ $L_{i}-M_{i}$. Switching the roles of $H$ and $H^{\prime}$, we see $D-D^{\prime} \sim M_{i}-L_{i}$. Finally, we may have $D-D^{\prime} \sim 0$.

(5) This is the mirror of the cases (1)-(3). 
Corollary 3.2. Suppose $D$ is the difference of two divisors appearing in the list (3-1). If $\operatorname{deg} D \geq-2$, then there is an exceptional line $E$ on $\bar{S}$ such that $D-E$ is also a difference of two divisors appearing in the list (3-1) and D.E $\geq-1$.

Proof. This is established through case-by-case analysis using Proposition 3.1 to look at all the possibilities for $D$.

A divisor $D$ on $\bar{S}$ is good if $h^{1}(D)=h^{2}(D)=0$.

Lemma 3.3. The divisors $-H$ and $-H^{\prime}$ on $\bar{S}$ are good.

Proof. The existence of the morphisms $f, f^{\prime}: \bar{S} \rightarrow \mathbb{P}_{\bar{F}}^{2}$ allow us to use the Leray spectral sequence. The arguments for $-H$ and $-H^{\prime}$ are the same so we only prove the result for $-H$.

Because $\bar{S}$ is a blowup of $\mathbb{P}_{\bar{F}}^{2}, f_{*} \mathcal{O}_{\bar{S}}=\mathcal{O}_{\mathbb{P}_{\bar{F}}^{2}}$ and $R^{j} f_{*} \mathcal{O}_{\bar{S}}=0$ if $j \geq 1$.

Since $\mathcal{O}_{\bar{S}}(-H) \cong f^{*} \mathcal{O}_{\mathbb{P}_{\bar{F}}^{2}}(-\ell)$, the projection formula gives

$$
\begin{aligned}
R^{j} f_{*} \mathcal{O}_{\bar{S}}(-H) & =R^{j} f_{*}\left(\mathcal{O}_{\bar{S}} \otimes f^{*} \mathcal{O}_{\mathbb{P}_{\bar{F}}^{2}}(-\ell)\right) \\
& \cong R^{j} f_{*} \mathcal{O}_{\bar{S}} \otimes \mathcal{O}_{\mathbb{P}_{\bar{F}}^{2}}(-\ell) \\
& \cong \begin{cases}\mathcal{O}_{\mathbb{P}_{\bar{F}}^{2}}(-\ell) & \text { if } j=0 \\
0 & \text { if } j \neq 0 .\end{cases}
\end{aligned}
$$

The Leray spectral sequence

$$
H^{i}\left(\mathbb{P}_{\bar{F}}^{2}, R^{j} f_{*} \mathcal{O}_{\bar{S}}(-H)\right) \Rightarrow H^{i+j}\left(\bar{S}, \mathcal{O}_{\bar{S}}(-H)\right)
$$

therefore degenerates to give

$$
H^{i}\left(\bar{S}, \mathcal{O}_{\bar{S}}(-H)\right) \cong H^{i}\left(\mathbb{P}_{\bar{F}}^{2}, \mathcal{O}_{\mathbb{P}^{2}}(-\ell)\right)
$$

for all $i$. The result follows because $H^{i}\left(\mathbb{P}_{\bar{F}}^{2}, \mathcal{O}_{\mathbb{P}^{2}}(-\ell)\right)=0$ for all $i$.

Lemma 3.4. Let $C$ be any divisor on $\bar{S}$, and let $E$ be one of the (-1)-curves. If $C-E$ is good and C.E $\geq-1$, then $C$ is good.

Proof. The long exact sequence in cohomology associated to

$$
0 \rightarrow \mathcal{O}_{\bar{S}}(C-E) \rightarrow \mathcal{O}_{\bar{S}}(C) \rightarrow \mathcal{O}_{E}(C) \rightarrow 0
$$

reads in part

$$
\begin{aligned}
& \longrightarrow H^{1}\left(\bar{S}, \mathcal{O}_{\bar{S}}(C-E)\right) \longrightarrow H^{1}\left(\bar{S}, \mathcal{O}_{\bar{S}}(C)\right) \longrightarrow H^{1}\left(\bar{S}, \mathcal{O}_{E}(C)\right) \longrightarrow \\
& \longrightarrow H^{2}\left(\bar{S}, \mathcal{O}_{\bar{S}}(C-E)\right) \longrightarrow H^{2}\left(\bar{S}, \mathcal{O}_{\bar{S}}(C)\right) \longrightarrow H^{2}\left(\bar{S}, \mathcal{O}_{E}(C)\right) .
\end{aligned}
$$

By hypothesis, the left-most term on each row is zero. The right-most term on each row is also zero because $H^{i}\left(\bar{S}, \mathcal{O}_{E}(C)\right) \cong H^{i}\left(\mathbb{P}_{\bar{F}}^{1}, \mathcal{O}_{\mathbb{P}^{1}}(C . E)\right)$. Hence $C$ is good. 


\section{The tilting Bundle $\mathcal{T}$}

In this section, we show that $\mathcal{T}$ is a tilting bundle and prove our main result.

Proposition 4.1. Let $i \geq 1$. Then $\operatorname{Ext}_{S}^{i}(\mathcal{T}, \mathcal{T})=0$.

Proof. By flat base change it suffices to prove this when $F$ is separably closed so we assume that $F=\bar{F}$. In that case $\operatorname{Ext}_{S}^{i}(\mathcal{T}, \mathcal{T})$ is isomorphic to a direct sum of terms of the form $H^{i}\left(S, \mathcal{O}_{S}\left(D-D^{\prime}\right)\right)$ where $D$ and $D^{\prime}$ are divisors in the list (3-1).

It therefore suffices to show that $D-D^{\prime}$ is good whenever whenever $D$ and $D^{\prime}$ are divisors in the list (3-1).

We argue by induction on $\operatorname{deg}\left(D-D^{\prime}\right)$. By Proposition $3.1-3 \leq \operatorname{deg}\left(D-D^{\prime}\right) \leq$ 3. If $\operatorname{deg}\left(D-D^{\prime}\right)=-3$, then $D-D^{\prime}$ is good by Lemma 3.3. Now suppose that $-2 \leq \operatorname{deg}\left(D-D^{\prime}\right) \leq 3$. By Corollary 3.2, there is an exceptional line $E$ such that $D-D^{\prime}-E$ is a difference of divisors in (3-1) and $\left(D-D^{\prime}\right) . E \geq-1$. By the induction hypothesis, $D-D^{\prime}-E$ is good, and it then follows from Lemma 3.4 that $D-D^{\prime}$ is good.

Lemma 4.2. Every $\mathcal{F} \in \operatorname{coh} \bar{S}$ has a finite resolution in which all terms are direct sums of invertible sheaves $\mathcal{O}_{\bar{S}}(D)$ for various divisors $D$ on $\bar{S}$.

Proof. Let $A$ be Cox's homogeneous coordinate ring for $\bar{S}[5$. Then $A$ is a polynomial ring with a grading by $\operatorname{Pic}(\bar{S})$. Let $M$ be a finitely generated graded $A$-module. Then $M$ has a finite projective resolution in the category of graded $A$ modules. By [8, Lemma 2.2], every finitely generated projective graded $A$-module is a direct sum of twists of $A$. The exact functor $\operatorname{Gr}(A, \operatorname{Pic}(\bar{S})) \rightarrow \mathrm{Q} \operatorname{coh} \bar{S}, M \rightsquigarrow \widetilde{M}$, described in [5. Thm. 3.11] sends the resolution of $M$ to an exact sequence in Qcoh $\bar{S}$ in which the right-most term is $\widetilde{M}$ and all other terms are direct sums of various $\mathcal{O}_{\bar{S}}(D), D \in \operatorname{Div}(\bar{S})$. Given $\mathcal{F} \in \operatorname{coh} \bar{S}$, there is a finitely generated graded $A$-module $M$ such that $\mathcal{F} \cong \widetilde{M}$.

For the rest of this paper, we will work in the derived category. If $\mathrm{C}$ is a triangulated category, we denote the shift of an object $\mathcal{M}$ by $\mathcal{M}[1]$. Recall that a subcategory of $\mathrm{C}$ is thick (epaisse) if it is closed under isomorphisms, shifts, taking cones of morphisms, and taking direct summands of objects.

Let $\mathrm{C}$ be a triangulated category and $\mathcal{E} \subset \mathrm{C}$. Then

- $\langle\mathcal{E}\rangle$ denotes the smallest thick triangulated subcategory of $\mathrm{C}$ containing $\mathcal{E}$;

- $\mathcal{E}^{\perp}$ denotes the full subcategory of $\mathcal{C}$ consisting of objects $\mathcal{M}$ such that $\operatorname{Hom}_{\mathcal{C}}(E[i], \mathcal{M})=0$, for all $E \in \mathcal{E}$;

and we say that

- $\mathcal{E}$ classically generates $\mathrm{C}$ if $\langle\mathcal{E}\rangle=\mathrm{C}$, and

- $\mathcal{E}$ generates $\mathrm{C}$ if $\mathcal{E}^{\perp}=0$.

Clearly if $\mathcal{E}$ classically generates $\mathcal{C}$, then it generates $\mathcal{C}$. Moreover, the converse is often true, due to a result of Ravenel and Neeman [7. We follow Bondal and Van den Bergh's restatement [4, Thm. 2.1.2].

Lemma 4.3. Let $\mathcal{T}$ be a compact object in a compactly generated triangulated category $\mathrm{C}$. Suppose $\mathcal{T}$ has the following property: if $\mathcal{M} \in \mathrm{C}$ and $\operatorname{Hom}_{\mathrm{C}}(\mathcal{T}, \mathcal{M}[n])=$ 0 for all $n$, then $\mathcal{M} \cong 0$. Then $\langle\mathcal{T}\rangle=\mathrm{C}$. In particular, if $\mathrm{C}$ is compactly generated and $\mathcal{T}$ generates $\mathrm{C}$, then $\mathcal{T}$ classically generates $\mathrm{C}$.

Theorem 4.4. $\overline{\mathcal{T}}$ generates and classically generates $\mathrm{D}^{b}(\operatorname{coh} \bar{S})$. 
Proof. It suffices to prove that $\overline{\mathcal{T}}$ classically generates $\mathrm{D}^{b}(\operatorname{coh} \bar{S})$. Since $\langle\operatorname{coh} \bar{S}\rangle=$ $\mathrm{D}^{b}(\operatorname{coh} \bar{S})$ it suffices to show that every coherent $\mathcal{O}_{\bar{S}}$-module belongs to $\langle\overline{\mathcal{T}}\rangle$.

If $D$ is an effective divisor on $\bar{S}$ we write $\mathcal{I}_{D}$ for the ideal vanishing on $D$ as a scheme. Thus $\mathcal{I}_{D} \cong \mathcal{O}_{\bar{S}}(-D)$. Whenever we write an arrow $\mathcal{O}_{\bar{S}}(-D) \rightarrow \mathcal{O}_{\bar{S}}$ it will be with the tacit understanding that this is the composition of an isomorphism $\mathcal{O}_{\bar{S}}(-D) \rightarrow \mathcal{I}_{D}$ followed by the inclusion $\mathcal{I}_{D} \rightarrow \mathcal{O}_{\bar{S}}$.

Since $M_{3} \cdot\left(L_{1}+M_{2}+M_{3}\right)=0$, therefore $\mathcal{O}_{M_{3}} \cong \mathcal{O}_{M_{3}}\left(L_{1}+M_{2}+M_{3}\right)$. It follows from the exact sequences

$$
0 \rightarrow \mathcal{O}_{\bar{S}}\left(L_{1}+M_{2}\right) \rightarrow \mathcal{O}_{\bar{S}}\left(L_{1}+M_{2}+M_{3}\right) \rightarrow \mathcal{O}_{M_{3}}\left(L_{1}+M_{2}+M_{3}\right) \rightarrow 0
$$

and

$$
0 \rightarrow \mathcal{O}_{\bar{S}}\left(-M_{3}\right) \rightarrow \mathcal{O}_{\bar{S}} \rightarrow \mathcal{O}_{M_{3}} \rightarrow 0
$$

that $\mathcal{O}_{M_{3}}$ and $\mathcal{O}_{\bar{S}}\left(-M_{3}\right)$ belong to $\langle\overline{\mathcal{T}}\rangle$. Hence $\mathcal{O}_{E}$ and $\mathcal{O}_{\bar{S}}(-E)$ belong to $\langle\mathcal{T}\rangle$ for all exceptional lines $E$.

Since $L_{i} \cdot L_{k}=0$ if $i \neq k$, there is an exact sequence

$$
0 \rightarrow \mathcal{O}_{\bar{S}}\left(-L_{i}-L_{k}\right) \rightarrow \mathcal{O}_{\bar{S}}\left(-L_{i}\right) \oplus \mathcal{O}_{\bar{S}}\left(-L_{k}\right) \rightarrow \mathcal{O}_{\bar{S}} \rightarrow 0 .
$$

Twisting by $L_{i}+M_{j}+L_{k}$, we obtain

$$
0 \rightarrow \mathcal{O}_{\bar{S}}\left(M_{j}\right) \rightarrow \mathcal{O}_{\bar{S}}\left(M_{j}+L_{k}\right) \oplus \mathcal{O}_{\bar{S}}\left(L_{i}+M_{j}\right) \rightarrow \mathcal{O}_{\bar{S}}\left(L_{i}+M_{j}+L_{k}\right) \rightarrow 0 .
$$

Therefore, $\mathcal{O}_{\bar{S}}\left(M_{j}\right) \in\langle\overline{\mathcal{T}}\rangle$. From the exact sequence

$$
0 \rightarrow \mathcal{O}_{\bar{S}} \rightarrow \mathcal{O}_{\bar{S}}\left(M_{j}\right) \rightarrow \mathcal{O}_{M_{j}}\left(M_{j}\right) \rightarrow 0,
$$

we deduce that $\mathcal{O}_{M_{j}}\left(M_{j}\right) \in\langle\overline{\mathcal{T}}\rangle$.

It follows that $\mathcal{O}_{E}(E) \in\langle\overline{\mathcal{T}}\rangle$ for every exceptional curve $E$. But $\mathcal{O}_{E}$ is also in $\langle\overline{\mathcal{T}}\rangle$ so, because $\mathrm{D}^{b}\left(\operatorname{coh} \mathbb{P}_{\bar{F}}^{1}\right)$ is generated by $\mathcal{O}_{\mathbb{P}_{\bar{F}}^{1}}$ and $\mathcal{O}_{\mathbb{P}_{\bar{F}}^{1}}(-1)$, it follows that $\mathrm{D}^{b}(\operatorname{coh} E) \subset\langle\overline{\mathcal{T}}\rangle$. Hence $\mathcal{O}_{E}(D) \in\langle\overline{\mathcal{T}}\rangle$ for all divisors $D$ on $\bar{S}$.

Suppose $\mathcal{O}_{\bar{S}}(D) \in\langle\overline{\mathcal{T}}\rangle$. Then $\mathcal{O}_{\bar{S}}(D-E) \in\langle\overline{\mathcal{T}}\rangle$ because there is an exact sequence

$$
0 \rightarrow \mathcal{O}_{\bar{S}}(D-E) \rightarrow \mathcal{O}_{\bar{S}}(D) \rightarrow \mathcal{O}_{E}(D) \rightarrow 0 .
$$

Likewise, $\mathcal{O}_{\bar{S}}(D+E) \in\langle\overline{\mathcal{T}}\rangle$ because there is an exact sequence

$$
0 \rightarrow \mathcal{O}_{\bar{S}}(D) \rightarrow \mathcal{O}_{\bar{S}}(D+E) \rightarrow \mathcal{O}_{E}(D+E) \rightarrow 0 .
$$

It follows that $\langle\overline{\mathcal{T}}\rangle$ contains $\mathcal{O}_{\bar{S}}(D)$ for all $D \in \operatorname{Div} \bar{S}$ and therefore, by Lemma 4.2 , contains $\mathcal{F}$ for every $\mathcal{F} \in \operatorname{coh} \bar{S}$.

When $F$ is not separably closed $\mathcal{T}$ need not split as a direct sum of line bundles so the arguments in Theorem 4.4 can not be used to prove directly that $\langle\mathcal{T}\rangle=\mathrm{D}^{b}(\operatorname{coh} S)$. We will instead show that $\mathcal{T}$ generates $\mathrm{D}^{b}(\operatorname{coh} S)$ and then apply Lemma 4.3 .

Theorem 4.5. Let $F$ be an arbitrary field. Then

$$
\mathrm{RHom}_{S}(\mathcal{T},-): \mathrm{D}^{b}(\operatorname{coh} S) \rightarrow \mathrm{D}^{b}(\bmod A)
$$

is an equivalence of categories.

Proof. By Proposition 4.1, $\operatorname{Ext}_{S}^{i}(\mathcal{T}, \mathcal{T})=0$ for $i>0$. By Proposition 2.2. $A=$ $\operatorname{End}_{S}(\mathcal{T})$ has finite global dimension. Further, we claim that $\mathcal{T}$ classically generates $\mathrm{D}^{b}(\operatorname{coh} S)$. Assuming this, the theorem follows directly from [2, Thm. 3.1.2].

So we need to prove that $\mathcal{T}$ classically generates $\mathrm{D}^{b}(\operatorname{coh} S)$. By Lemma 4.3 it suffices to show that if $\mathcal{M} \in \mathrm{D}^{b}(\operatorname{coh} S)$ and $\operatorname{RHom}_{S}(\mathcal{T}, \mathcal{M})=0$, then $\mathcal{M}=0$. 
Suppose that $\mathcal{M} \in \mathrm{D}^{b}(\operatorname{coh} S)$ and $\operatorname{RHom}_{S}(\mathcal{T}, \mathcal{M})=0$. Consider the cartesian square

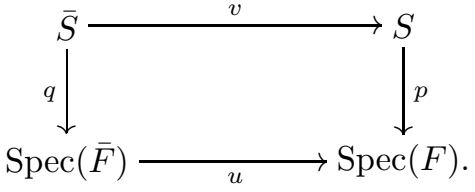

Since $u$ (and therefore $v$ ) is flat, the natural transformation

$$
u^{*} R f_{*} \rightarrow R g_{*} v^{*}
$$

is an isomorphism [6, (3.18)]. We now have

$$
\begin{aligned}
0=u^{*} \operatorname{RHom}_{S}(\mathcal{T}, \mathcal{M}) & \cong u^{*} R p_{*} \operatorname{RHom}_{S}(\mathcal{T}, \mathcal{M}) \\
& \cong R q_{*} v^{*} \operatorname{RHom}{ }_{S}(\mathcal{T}, \mathcal{M}) \\
& \cong R q_{*} v^{*}\left(\mathcal{T}^{\vee} \otimes_{S}^{L} \mathcal{M}\right) \\
& \cong R q_{*}\left(\overline{\mathcal{T}}^{\vee} \otimes_{\bar{S}}^{L} L v^{*} \mathcal{M}\right) \\
& \cong R q_{*} \operatorname{RH}_{\bar{S}}\left(\overline{\mathcal{T}}, L v^{*} \mathcal{M}\right) \\
& \cong \operatorname{RHom}_{\bar{S}}\left(\overline{\mathcal{T}}, L v^{*} \mathcal{M}\right) .
\end{aligned}
$$

But $\overline{\mathcal{T}}$ classically generates and therefore generates $\mathrm{D}^{b}(\operatorname{coh} \bar{S})$, so it follows that $v^{*} \mathcal{M}=0$. Since $v^{*}$ is faithful, $\mathcal{M}=0$, and we are done.

\section{REFERENCES}

[1] M. Auslander, I. Reiten, and S. Smalo, Representation Thoery of Artin Algebras, Camb. Studies in Adv. Math., No. 36, 1995.

[2] D. Baer, Tilting sheaves in representation theory of algebras, Manus. Math., 60 (1988) 323347.

[3] M. Blunk, Del Pezzo surfaces of degree 6 over an arbitrary field, J. Alg., (to appear), arXiv:08050119v2.

[4] A. Bondal and M. Van den Bergh, Generators and representability of functors in commutative and noncommutative geometry, Moscow Mathematical Journal, 3 (2003) 1-36.

[5] D.A. Cox, The homogeneous coordinate ring of a toric variety, J. Alg. Geom., 4 (1995) 17-50.

[6] D. Huybrechts, Fourier-Mukai transforms in algebraic geometry, Oxford Univ. Press, 2006.

[7] A. Neeman, The connection between the K-theory localization theorem of Thomason, Trobaugh and Yao and the smashing subcategories of Bousfield and Ravanel, Ann. Sci. de l' Éc. Norm. Sup., 25 (1992) 547-566.

[8] S.P. Smith, The Grothendieck and Picard groups of a toric DM stack, arXiv:0806.0192 2.

Department of Mathematics, Univ. of British Columbia., Department of Mathematics, Box 354350, Univ. Washington, Seattle, WA 98195

E-mail address: mblunk@math.ubc.ca, sjsierra@math.washington.edu, smith@math.washington.edu 